\title{
Novel Agents that Prevent Atherosclerosis: Multivalent Peptide Constructs and Self-Assembling Cyclic DL- $\alpha$-Peptides
}

\author{
Bruce E. Maryanoff ${ }^{1}$, Luke J. Leman'1, Yannan Zhao', Tomohiro Imura1, \\ Audrey S. Black ${ }^{1}$, David J. Bonnet ${ }^{2}$, Linda K. Curtiss ${ }^{2}$, \\ and M. Reza Ghadiri, ${ }^{1,3}$,* \\ ${ }^{I}$ Department of Chemistry; ${ }^{2}$ Department of Immunology and Microbial Science; ${ }^{3}$ The Skaggs Institute for \\ Chemical Biology, The Scripps Research Institute, La Jolla, CA, 92037, USA
}

\section{Introduction}

Heart disease is the number one killer worldwide, and is responsible for nearly a third of all deaths in the USA. The principal cause of heart disease is lipid accumulation in the arteries with attendant deposition of atherosclerotic plaque, which is comprised mainly of lipids, cholesterol, calcium salts, cells, and cellular debris. Atherosclerosis can be treated by reducing low-density lipoproteincholesterol (LDL-C) via administration of statin drugs. High-density lipoproteins (HDLs) appear to protect against atherosclerosis, such that an increase in their levels and function is desirable. HDL particles, ranging in size from 7-13 nm, are in a constant state of dynamic flux, where the large particles convert into smaller particles, or release free apoA-I. HDL particles accept cholesterol and lipids from peripheral cells and plaques for elimination in the liver (Reverse Cholesterol Transport). The antiatherogenic action of HDL is related to the major structural protein, apolipoprotein A-I (apoA-I), a 243-mer in humans that is comprised of 10 amphiphilic $\alpha$-helices [1]. ApoA-I mimetic peptides are generally amphiphilic $\alpha$-helices that can boost levels of cholesterol-mobilizing, HDL-like particles or improve the functional properties of such particles [1]. We have been involved in the design, synthesis, and functional characterization of novel molecules that mimic apoA-I based on the multivalent presentation of 23-mer and 16-mer amphiphilic, $\alpha$-helical peptides [2]. Some of these derivatives were athero-preventive in a key mouse model on i.p. and oral (!) administration [3]. Results from this work led us to explore amphiphilic cyclic DL- $\alpha$-peptides that could self-assemble into amphiphilic nanotubes.

\section{Results and Discussion}

We utilized native chemical ligation [4] to link amphiphilic peptides to small-molecule scaffolds that possessed 2, 3, or 4 reactive arms to obtain two families of monomer, dimer, trimer, and tetramer peptide constructs based on amphiphilic, $\alpha$-helical subunits containing 23 or 16 amino acids [2]. These multivalent constructs were combined, in a formulation, with $(R)-(+)-1,2$-dimyristoyl-sn-glycero-3phosphocholine (DMPC) to obtain discoidal nanoparticles in the HDL size range [2]. We systematically studied the formation of lipid nanodiscs and their functional effects in relation to helix multimerization. For in vitro functional characterization, we measured the effect of each construct on cholesterol efflux from cholesterol-laden macrophage cells (J774). In both series, the multivalent constructs promoted cholesterol efflux more efficiently than the monomeric peptide. In the 16-mer family, the efficacy was in the order: tetramer-16 > trimer-16> dimer-16 >> monomer-16. All of the peptide nanoparticles, except for monomer-16, fostered HDL remodeling in human plasma in vitro, which constitutes to the increase in small, dense HDL levels within the HDL particle distribution.

When administered i.p. to mice, the nanoparticles containing multivalent constructs exhibited superior plasma concentrations and much longer residence times (e.g., 6-8 h for trimer-23 and trimer16 nanoparticles) compared with nanolipid particles from monomers. The small, dense HDL bands were enhanced from 2-8 h post-injection for dimer-23 and trimer-23, reflecting substantial remodeling of mouse HDLs in vivo. We carried out athero-prevention studies in low-density lipoprotein receptornull (LDLr-/-) mice. At 10 weeks of age, the mice were switched from a normal chow diet to a highfat diet, and daily 40-mg/kg i.p. injections of trimer-23/DMPC nanoparticles were initiated. After two weeks, plasma total cholesterol levels were reduced by $40 \%$, compared to vehicle (phosphate-buffered saline, PBS) controls. The plasma HDLs were also remodeled in favor of smaller HDL particle sizes. In further cholesterol reduction studies, trimer-23/DMPC nanoparticles were administered orally in the drinking water $(\sim 50 \mathrm{mg} / \mathrm{kg} /$ day $)$. After two weeks, the trimer nanoparticles reduced plasma total 
cholesterol levels by $40 \%$ compared to PBS control. To establish the effect of the trimer-23/DMPC nanoparticles on atherosclerotic plaque development, groups of LDLr-/- mice were treated with daily i.p. injections $(40 \mathrm{mg} / \mathrm{kg}$ ) or orally (in drinking water, $\sim 50 \mathrm{mg} / \mathrm{kg} /$ day) for 10 weeks. Daily i.p. treatment with the trimer-23 nanoparticles reduced aorta lesion areas and heart aortic valve plaque volumes by $55 \%$ and $61 \%$, respectively. Surprisingly, oral administration of the trimer-23 nanoparticles for 10 weeks markedly reduced aorta lesion areas and heart aortic valve plaque by $50 \%$ and $70 \%$.

These exciting findings inspired us to consider the potential for athero-preventive effects with amphiphilic cyclic DL- $\alpha$-peptides, a signature molecule of the Ghadiri group, which ought to selfassemble into amphiphilic nanotubes with dimensionality that might mimic apoA-I $\alpha$-helices (Figures 1 and 2). The synthesis of amphipathic cyclic DL- $\alpha$-peptides involved efficient assembly of linear precursor peptides and cyclization on a solid support. Certain cyclic peptides promoted cellular cholesterol efflux in vitro and HDL remodeling in vivo; $c$ [wLwReQeR], $\mathbf{1}$, and $c$ [wLwSeQsO], 2, were prototypes (Figure 2). $N$-Methylation of L and Q in 2, which will prevent nanotube assembly, markedly attenuated cholesterol efflux activity. Compound $\mathbf{1}$ was administered orally (in drinking water ad libitum) to LDLr-/- mice on a high-fat diet for 10 weeks with two separate dose regimes: 35 and $3.5 \mathrm{mg} / \mathrm{kg}$. At 2 and 10 weeks, 1 (at $35 \mathrm{mg} / \mathrm{kg}$ ) reduced total plasma cholesterol by $54 \%$ and $30 \%$, respectively (vs. PBS control). After 10 weeks, 1 (at $35 \mathrm{mg} / \mathrm{kg}$ ) was found to reduce aortic lesion area by $26 \%$ and aortic valve plaque by $50 \%$ (vs. PBS). The dose of $3.5 \mathrm{mg} / \mathrm{kg}$ was not effective. Numerous cyclic DL- $\alpha$-peptides with different side chains were evaluated in vitro to find those with promise for oral testing in vivo with an endpoint of cholesterol lowering after oral administration for 2 weeks (at $35 \mathrm{mg} / \mathrm{kg}$ ). Compound 1 exhibited the best activity (-50\%), with no activity evident for $\mathbf{1}$ at one-tenth of the $35-\mathrm{mg} / \mathrm{kg}$ dose. Other effective compounds were: $c$ [wLwSeQhK] $(-33 \%)$ and $c$ [wLwKhShK] $(-31 \%)$.

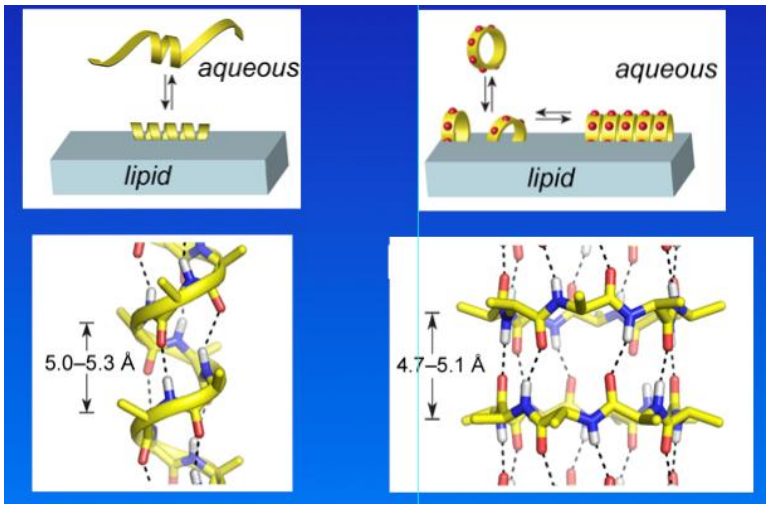

Fig. 1. Comparison of $\alpha$-helix and DL- $\alpha$-peptides.

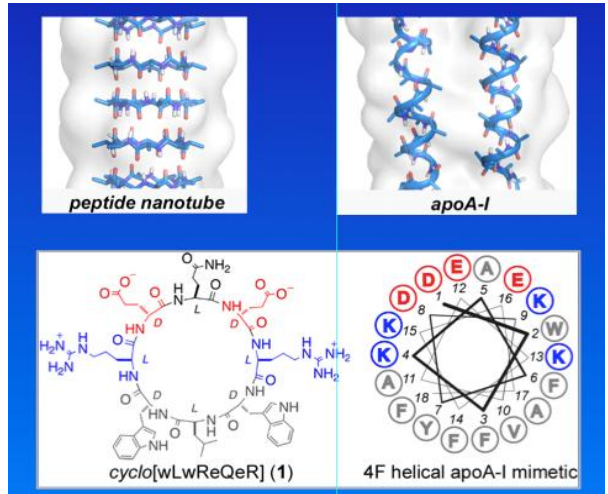

Fig. 2. Formation of nanotubes.

We used DNA sequencing and qPCR of group-specific 16S rRNA gene copies vs. total bacterial 16S rRNA gene copies to probe the mechanistic relevance of the microbiome (Figure 3). Compound 1 modulated the microbiome such that the profile in mice fed a high-fat diet was shifted to one akin to that in mice fed a chow diet (Figure 3). Specifically, $c$ [wLwReQeR] and $c$ [wLwKhShK] decreased the ratio of firmicutes/bacteroidetes by $56 \%$ and $35 \%$, respectively (Figure 4 ). These results suggest that a key factor in the observed oral activity for these cyclic peptides may well be modulation of the mouse microbiome. In conclusion, certain cyclic DL- $\alpha$-peptides may offer a novel means for treating atherosclerosis. 


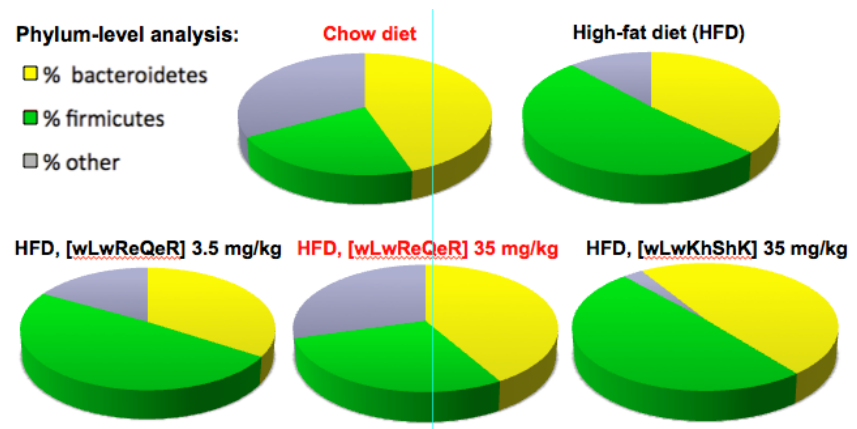

Fig. 3. Microbiome profiles in tested mice.

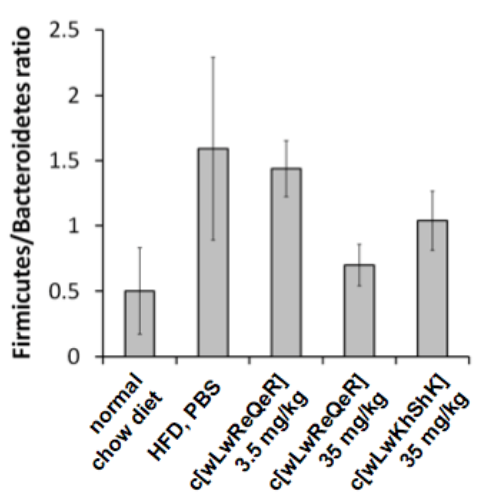

Fig. 4. Ratio of firmicutes/bacteroidetes.

\section{Acknowledgments}

We thank the NIH for financial support (NHLBI HL104462), the American Heart Association Western States Affiliate for a postdoctoral fellowship (12POST12040298) to Dr. Y. Zhao, and the National Institute of Advanced Science and Technology (AIST) for a visiting research fellowship to Dr. T. Imura.

\section{References}

1.Leman, L.J., Maryanoff, B.E., Ghadiri, M.R. J. Med. Chem. 57, 2169-2196 (2014), http://dx.doi.org/10.1021/jm4005847

2.Zhao, Y., Imura, T., Leman, L.J., Maryanoff, B.E., Ghadiri, M.R. J. Am. Chem. Soc. 135, 13414-13424 (2013), http://dx.doi.org/10.1021/ja404714a

3.Zhao, Y., Black, A.S., Bonnet, D.J., Maryanoff, B.E., Curtiss, L.K., Leman, L.J., Ghadiri, M.R. J. Lipid Res. 55, 2053-2063 (2014), http://dx.doi.org/10.1194/jlr.M049262

4.Dawson, P.E., Muir, T.W., Clark-Lewis, I., Kent, S.B. Science 266, 776-779 (1994). 